\title{
Kidney transplantation during a twin pregnancy. Case report and review of the literature
}

\author{
Jorge Vega $\cdot$ Christian Videla $\cdot$ Oscar Santis · \\ Mauricio Lira $\cdot$ Helmuth Goecke
}

Received: 27 September 2011 / Accepted: 6 March 2012/Published online: 11 April 2012

(C) Japanese Society of Nephrology 2012

\begin{abstract}
Kidney transplant in a pregnant woman is exceptional, with only six cases being reported. Pregnancy was not known at the time of the transplant in five of these cases. We report the case of a 26-year-old woman who was diagnosed as carrying a twin pregnancy 4 months after starting hemodialysis. In order to improve the survival chances for the twins, she underwent an orthotopic renal transplant from a living donor at 20 weeks of gestation. The allograft functioned immediately and 4 weeks passed without incident. At the 26th week of gestation, the patient had a precipitous labor, delivering two male babies with no malformations, weighing 755 and $890 \mathrm{~g}$, who died due to respiratory failure. The allograft worked normally afterwards. Sixteen months later, the patient delivered a normal 37 weeks' gestation baby. The renal graft continued working normally for the next 20 years, after which signs of chronic allograft nephropathy developed. Azathioprine was replaced with mycophenolate mofetil. At the last evaluation, 23 years after transplant, the patient's serum
\end{abstract}

J. Vega $\cdot$ C. Videla

Servicio de Medicina Interna, Sección Nefrología, Diálisis y

Trasplante Renal, Hospital Dr. Gustavo Fricke, Viña del Mar, Chile

J. Vega $(\bowtie) \cdot$ H. Goecke

Escuela de Medicina, Universidad de Valparaíso, 5 Norte 1035, 2541579 Viña del Mar, V Región, Chile

e-mail: jvegastieb@gmail.com

J. Vega $\cdot$ H. Goecke

Servicio de Medicina Interna, Sección Nefrología, Diálisis y

Trasplante Renal, Hospital Naval A. Nef, Viña del Mar, Chile

O. Santis · M. Lira

Servicio de Urología, Hospital Dr. Gustavo Fricke, Viña del Mar, Chile creatinine was $2.9 \mathrm{mg} / \mathrm{dl}$ and her estimated glomerular filtration rate (eGFR) was $22 \mathrm{ml} / \mathrm{min} / 1.73 \mathrm{~m}^{2}$. This unique case demonstrates that it is possible to perform an orthotopic kidney transplant in a 20 -week twin-gestation, with a long graft survival time.

Keywords Pregnancy, multiple $\cdot$ Kidney transplantation . Allogeneic transplantation - Cyclosporine - Azathioprine · Mycophenolate mofetil

\section{Introduction}

The probability of pregnancy in a patient on hemodialysis (HD) is low, given the marked reduction in the fertility of women with kidney failure. However, pregnancy occurs in approximately $1 \%$ of patients undergoing dialysis, usually within the first several years of starting the procedure. Although $42 \%$ of women receiving HD who are of childbearing age continue menstruating, many of their cycles are likely anovulatory [1]. The diagnosis of pregnancy in HD patients is often difficult, because levels of beta-human chorionic gonadotropin (b-hCG) are usually elevated in women receiving dialysis; therefore, when pregnancy is suspected, ultrasonography should be performed. Pregnancy is generally not advisable for patients on HD, as the fetal outcome is poor. Only $23-55 \%$ of pregnancies result in live infants; of these, $85 \%$ are born premature and $28 \%$ are born small for gestational age [2,3]. Maternal morbidity is also high, and arterial hypertension worsens in more than $80 \%$ of pregnant women on dialysis, sometimes even resulting in maternal deaths $[2,4]$. The management of a pregnant patient on HD includes the increase in the frequency and duration of dialysis sessions, up to 6 days per week, attempting to keep the blood urea nitrogen 
(BUN) below $50 \mathrm{mg} / \mathrm{dl}$, which may avoid polyhydramnios, control hypertension, and improve the mother's nutritional status [2, 3, 5]. It has also been recommended to use erythropoietin and to place the patient on a kidney transplant (KTx) list, given that successful KTx markedly improves fetal prognosis [2]. This last recommendation is based only on six case reports [6-12]. In five of these, pregnancy was not known at the time of KTx and in the other case, it was suspected [10].

This communication, to the best of our knowledge, is the first KTx performed intentionally on a woman carrying a confirmed pregnancy, as well as the first performed on a patient bearing a twin pregnancy, and in an orthotopic position.

\section{Case report}

A woman who had mesangiocapillary glomerulonephritis diagnosed when she was 6 years old had a pregnancy at age 20 years, which was terminated by cesarean section; the newborn was healthy and followed a normal development. At age 26 years, the patient developed a uremic syndrome (BUN $169 \mathrm{mg} / \mathrm{dl}$, serum creatinine $17 \mathrm{mg} / \mathrm{dl}$ ). She had not had a medical checkup during the intervening 6 years. The patient started HD in August, 1987.

In October, 1987, she was evaluated by an obstetrician because of menometrorrhagia and severe anemia (hematocrit $13 \%$ ), with a normal gynecological examination. The upper gastrointestinal endoscopy showed mild antral gastritis and a bone marrow smear revealed erythroblastic hyperplasia with slight dyserythropoiesis. Ferrous sulphate, folic acid, and vitamins were prescribed.

Four months after the initiation of HD, due to a menstrual delay of 2 weeks associated with vomiting, her physician requested a pregnancy test, which was positive. It was thought that the pregnancy had little chance to succeed and miscarriage was expected soon. The patient continued on HD, $4.5 \mathrm{~h}$ per session, three times a week, using methyldopa for hypertension. Because of progressive anemia (hematocrit $12 \%$ ), she received 6 units of red blood cells.

One month after diagnosing the pregnancy, an ultrasound revealed an approximately 10 weeks' gestation of two fetuses. Ultrasound was repeated after 5 weeks, confirming two active fetuses, with a posterior placenta and normal amniotic fluid. The gestational age was estimated to be 17 weeks.

Her sister, with whom she had no HLA mismatches, offered to donate a kidney to save the twins, and the patient accepted. The case was presented to the ethics committee of the hospital, which authorized the KTx, considering that it was ethically irreproachable to try to save the twins, who had little probability of survival if the mother remained on dialysis. At the time, there was only one published report of a KTx on a patient who was already pregnant (at 12 weeks' gestation), which had a good outcome for both the mother and the child.

On March 17 1988, at the 18th week of gestation, she was admitted to the obstetric unit in order to prepare her for kidney transplantation. She was anemic (hematocrit $16 \%$, hemoglobin $5.5 \mathrm{~g} / \mathrm{dl}$ ) and malnourished (body mass index [BMI] $19 \mathrm{~kg} / \mathrm{m}^{2}$, serum albumin $3.2 \mathrm{~g} / \mathrm{dl}$ ). She remained on hemodialysis for $4.5 \mathrm{~h}$ each session and her BUN ranged from 60 to $86 \mathrm{mg} / \mathrm{dl}$. At the 19 th gestational week, the sonography showed both twins in shoulder presentation, their biparietal diameters (BPD) were 46 and $47 \mathrm{~mm}$, abdominal circumferences 45 and $46 \mathrm{~mm}$, femur lengths 28 and $28 \mathrm{~mm}$, tibias 24 and $25 \mathrm{~mm}$, and radiuses 25 and $25 \mathrm{~mm}$. The placenta was biamniotic, in the posterior position, and there was moderate polyhydramnios. It was concluded that fetal development was adequate. Fenoterol was used to avoid uterine contractions.

The patient was given three whole blood transfusions from her sister, along with $100 \mathrm{mg}$ of azathioprine (AZA), at intervals of 15 days each, to reduce the possibility of rejection of the graft during the pregnancy, given the high titer of antibodies against lymphocyte panel.

At 20 weeks of gestation (March 29, 1988), after administrating $150 \mathrm{mg}$ progesterone and $1 \mathrm{~g}$ of methylprednisolone (MPDN), an orthotopic KTx was performed on the left flank, since the size of the abdomen did not allow localizing the kidney in the usual position. The renal artery of the graft was anastomosed to the aorta, at the origin of its own renal artery; the renal vein of the graft was anastomosed to the renal vein of the patient and the renal pelvis of the graft to the renal pelvis of the recipient. A nephrostomy was left for protection. General anesthesia with halothane and epidural anesthesia with bupivacaine was used. The reactive antibodies against a panel of lymphocytes was $45 \%$ in the days previous to the surgery and the cross-match between the donor and the recipient was negative. Immunosuppression was continued with $100 \mathrm{mg}$ of AZA with the addition of $60 \mathrm{mg}$ of prednisone (PDN), antibiotic prophylaxis with cefazolin, and antihypertensive therapy with hydralazine and methyldopa. The graft started to function immediately and azotemia decreased rapidly. On the sixth post-transplant day, the patient's BUN was $14.5 \mathrm{mg} / \mathrm{dl}$ and her serum creatinine was $1.1 \mathrm{mg} / \mathrm{dl}$. One day after transplant, uterine contractions developed, which were controlled with fenoterol. On the fifth day post-KTx, jaundice was noted, associated with pruritus and slight elevation of aminotransferases, which were attributed to a cholestasis associated to methyldopa, which was withdrawn. In the following days, the hepatic disorder disappeared. On the sixth day post-KTx, an ultrasound showed 
both fetuses in good condition and discrete polyhydramnios. Oral fenoterol to avoid uterine contractions and weekly progesterone $(150 \mathrm{mg})$ was used. Twelve days after transplantation, ultrasound showed normal fetal movements. One fetus was in cephalic and the other in breech presentation. Their measurements at that moment were: BPD 56 and $58 \mathrm{~mm}$, femur length 38 and $39 \mathrm{~mm}$, and abdominal circumference 65 and $65 \mathrm{~mm}$. The gestational age was estimated to be $22+5$ weeks. At the 30 th day after transplantation, a new ultrasound revealed increased amniotic fluid and live fetuses. The BPDs were 64 and $64 \mathrm{~mm}$, femur length 43 and $43 \mathrm{~mm}$, abdominal circumference 70 and $64 \mathrm{~mm}$, and there were two amniotic sacs. The gestational age was estimated to be $25+3$ weeks. It was stated that one of the fetuses had reduced its abdominal circumference.

The patient remained hospitalized without complications and 1 month after transplant, she was authorized to visit her home for a weekend. She returned 2 days later in labor, which could not be stopped. At 32 days post-transplant and at 25 and 5/7 weeks of gestation, the patient delivered vaginally two males babies of birth weight 890 and $755 \mathrm{~g}$ who had an Apgar test of 1 after one min and 6 after $5 \mathrm{~min}$. The first twin was born by cephalic delivery and the second by incomplete breech delivery. Their lengths were 35 and $32 \mathrm{~cm}$; both had a cranial circumference of $25 \mathrm{~cm}$. The twins were both intubated and connected to oxygen. They were not ventilated because, at that moment, there were no available mechanical ventilators at the neonatology unit. Both infants died $4 \mathrm{~h}$ later due to respiratory failure. The amniotic fluid contained meconium, indicating the presence of fetal distress. In the following days, the patient developed endometritis, which responded to antibiotics. She was discharged 43 days postKTx with normal kidney function (serum creatinine $0.60 \mathrm{mg} / \mathrm{dl}$, creatinine clearance $115 \mathrm{ml} / \mathrm{min} / 1.73 \mathrm{~m}^{2}$ ).

Seven months after the KTx, and while undergoing treatment with AZA $125 \mathrm{mg}$, PDN $10 \mathrm{mg}$, and nitrendipine $20 \mathrm{mg}$ daily, a new pregnancy was diagnosed. Sixteen months post-KTx, she gave birth by cesarean section to a healthy baby of 37 weeks' gestation who weighed 3,240 g. One year later, she underwent tubal ligation by laparoscopy.

In the first 5 years after KTx, the allograft functioned normally (serum creatinine $0.9 \mathrm{mg} / \mathrm{dl}$, estimated glomerular filtration rate ([GFR] by Cockcroft-Gault $136 \mathrm{ml} / \mathrm{min}$ ); the only intercurrent conditions were one episode of lower urinary tract infection and shingles. Ten years after KTx, her renal function was adequate (serum creatinine $1.37 \mathrm{mg} / \mathrm{dl}$, eGFR $68 \mathrm{ml} / \mathrm{min}$ ), and the only complication was a duodenal ulcer that was treated with ranitidine. Twenty years after transplantation, proteinuria was detected $(1,452 \mathrm{mg} /$ $24 \mathrm{~h}$ ) and the graft function began to deteriorate slowly (serum creatinine $2.6 \mathrm{mg} / \mathrm{dl}$, eGFR $31 \mathrm{ml} / \mathrm{min}$ ), which was attributed to a chronic allograft nephropathy. It was decided not to perform a renal biopsy, but, instead, to change AZA to mycophenolate mofetil (MMF) $1,250 \mathrm{mg} /$ day; this was associated with the stabilization of kidney function. In the most recent visit, 23 years after KTx, the patient's serum creatinine was $2.9 \mathrm{mg} / \mathrm{dl}$ and her eGFR was $22 \mathrm{ml} /$ $\min$.

\section{Discussion}

The first KTx on a pregnant woman was performed in 1979 [8]. To date, six transplants have been performed during pregnancy and published in the literature: five of them during undiagnosed pregnancies and one in which there was suspicion of pregnancy prior to transplantation [6-12]. Four women were nulliparous at the time of transplantation, two were second transplants, and three were performed using live donors. The age of the recipients ranged from 18 to 36 years, and time spent on dialysis ranged from 5 months to several years. Three of the six pregnancies were discovered by accident during routine ultrasound assessment of the kidney graft and one studying a painful abdominal mass. Pregnancy diagnosis was made between day 5 and 100 after kidney transplant. Gestational age at the time of transplant varied from 10 to 13 weeks. Vaginal delivery was performed in four births and cesarean section in two. All the newborns were premature, with weights ranging from 870 to $2,948 \mathrm{~g}$. Four of them presented perinatal complications, secondary to prematurity (hyperbilirubinemia, respiratory distress due to hyaline membrane disease, and gastroesophageal reflux).

Three newborns presented congenital malformations. One of them was exposed to MMF and tacrolimus since the 6th week, presenting finger and nail hypoplasia and bilateral shortening of the fifth fingers. There is one previous communication in humans of this malformation with the use of MMF, being categorized as $\mathrm{C}$ drug during pregnancy, so it is possible to think that MMF may have had a causal role in the defects observed [13]. In animal teratogenicity assays, head and eye malformations as well as diaphragmatic hernias have been described [14-16]. In humans, microtia, auditory canal atresia, cleft lip and palate, micrognathia, hypertelorism, diaphragmatic hernia, and ocular coloboma are related to MMF use [13, 16]. Digital malformations have been related to alcohol and phenytoin use during pregnancy in humans and nifedipine in animals; nevertheless, this patient had not received any of them. Tacrolimus is considered to be a safe drug to use during pregnancy, without presenting any increase in malformation rate with its use. There are no digital defects related with this drug [17-19]. Another infant had two 


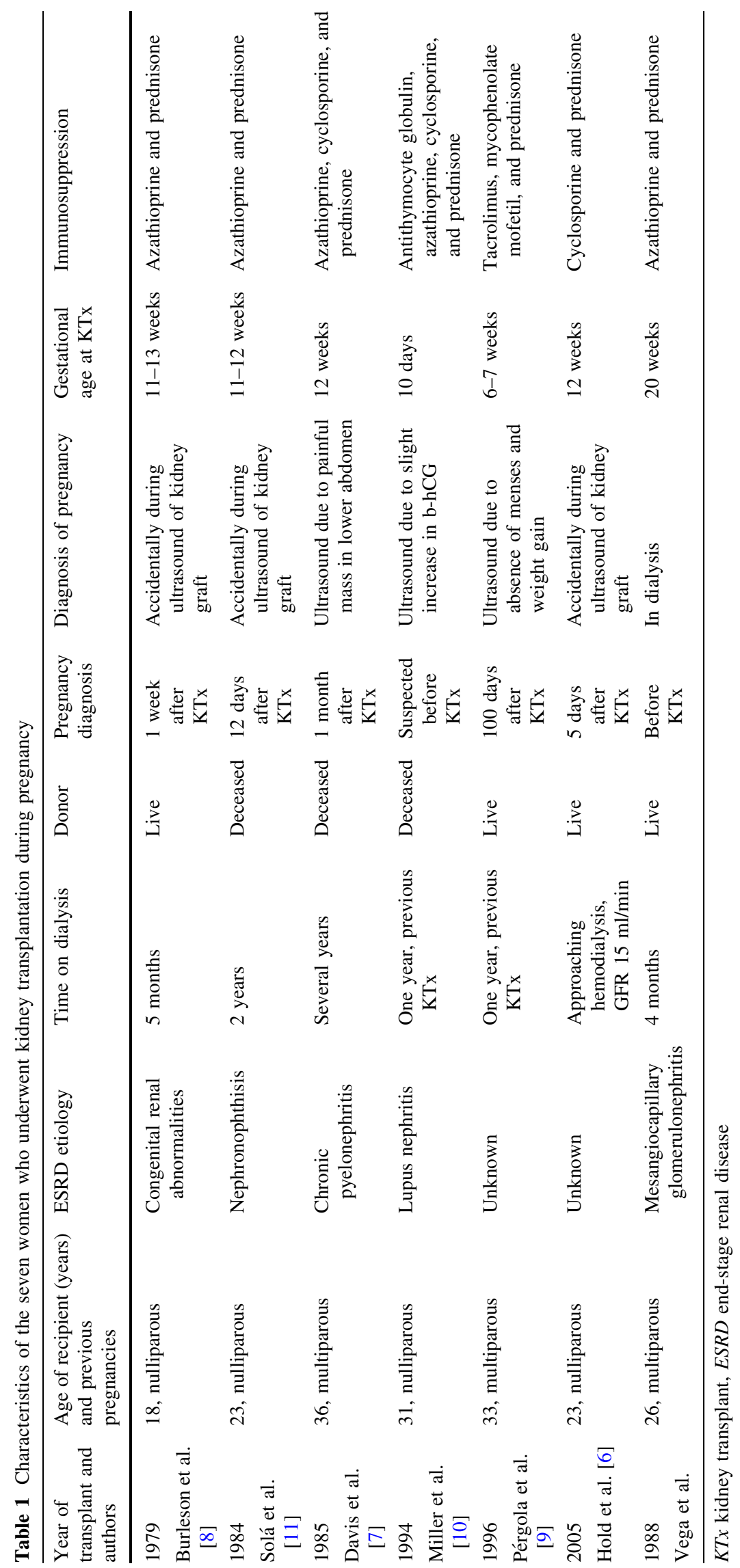




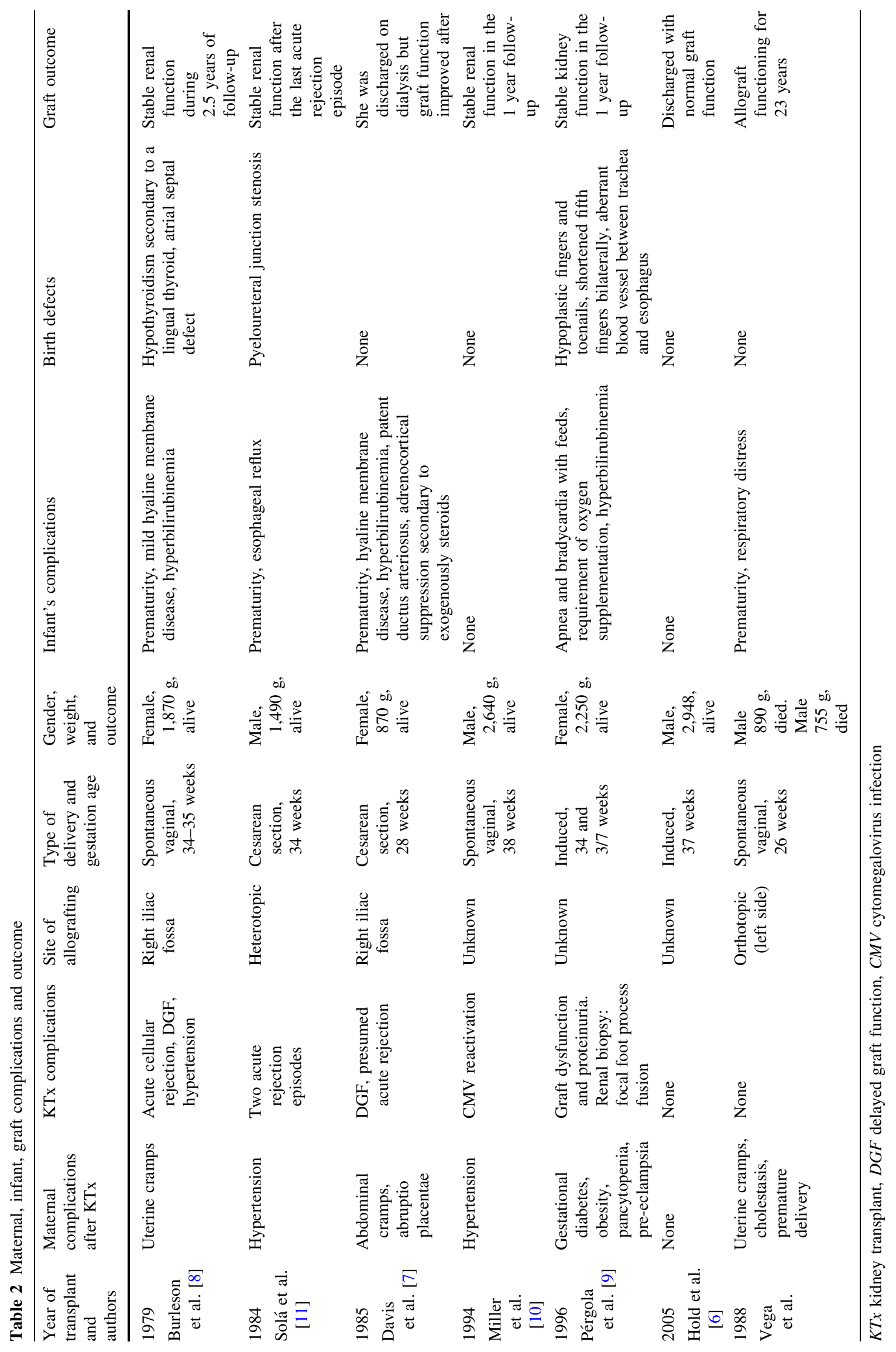


development defects: lingual thyroid and interatrial septum defect, and the third infant a pyeloureteral junction stenosis. It is unlikely that the immunosuppression had a role in the defects of the two infants because transplantation was performed between weeks 11 and 13, knowing that organogenesis is almost completed at week 12 [8, 11, 12]. Uremic milieu of both patients may have participated in the occurrence of malformations. All the infants resulting from these six transplants during pregnancy survived. Maternal complications more frequently observed after kidney transplantation were hypertension and acute cellular rejection. Four patients received azathioprine, three cyclosporine, and one patient tacrolimus and MMF. Only one case was induced with antithymocyte globulin (ATG), during a second transplant. The most relevant data of these six case reports are shown in Tables 1 and 2.

Current recommendations suggest that women who undergo any type of organ transplant wait at least 18-24 months after surgery to become pregnant. This allows the graft time to stabilize its function and immunosuppression to achieve appropriate maintenance levels [20].

This clinical case shows that is possible to perform an orthotopic KTx during a twin pregnancy at 20 weeks of gestation, allowing it to continue for almost 6 weeks and obtaining neonates with a weight that, today, 23 years later, with the available technology, would have had a high probability of survival. This case also demonstrates that it is possible to have a long survival time for a kidney allograft with immunosuppression using AZA and corticoids, in spite of higher titers of antibodies against a panel of lymphocytes when the KTx is performed with a live donor during pregnancy, after performing donor-specific transfusions. It is also possible that the natural immunosuppression induced by pregnancy had an influence in the long survival time of the kidney graft in this patient.

It is not our intention to recommend a kidney transplant in pregnant women on dialysis. We just wanted to communicate an experience of almost a quarter century ago, when we performed a kidney transplant in a woman carrying a twin pregnancy because, at that time, it seemed to be the best alternative in order to result in viable twins.

During the 1980s, conception of a dialysis patient had only a $20 \%$ chance of success. Severe transfusion-dependent anemia, polyhydramnios (due to fetal osmotic diuresis induced by elevated placental BUN), elevated risk of premature rupture of membranes of a twin pregnancy, hypertension, and acute extracellular volume changes and hypotension during dialysis sessions with compromised utero-placental circulation made it extremely difficult for the pregnancy to achieve viable fetuses [21]. This lead to the acceptance of the patient's sister's proposition of donating a kidney with the intention to try to save the twins. Successful transplantation would help to avoid the uremic milieu surrounding the twins' development, correct the anemia, and avoid hemodynamic events during dialysis.

Several transplant specialists were gathered in a meeting to discuss the risks of performing an orthotopic transplant on a malnourished, anemic, and hypersensitized pregnant patient against the potential benefits of a successful transplant. The decision to proceed with the transplantation was the choice of the group. Today, with the knowledge that dialysis six times a week in an attempt to be sure of reaching $20 \mathrm{~h} /$ week or nocturnal dialysis, to keep BUN levels less than $50 \mathrm{mg} / \mathrm{dl}$ with blood pressure tightly controlled with ultrafiltration and medications, fetal monitoring during dialysis, and the judicious use of erythropoietin, it is possible to increase baby survival up to $75-80 \%$ in pregnant women on dialysis $[1-3,22]$. We certainly would not decide to perform a kidney transplant as the first choice.

\section{References}

1. Stratilatova M. Pregnancy in ESRD and transplant patients. http://www.aakp.org/aakp-library/pregnancy-esrd-transplant-patients/. Accessed 9 Dec 2011.

2. Krane NK. Renal disease and pregnancy. http://emedicine. medscape.com/article/246123-overview. Accessed 27 Jun 2011.

3. Hou S. Pregnancy in women treated with dialysis: lessons from a large series over 20 years. Am J Kidney Dis. 2010;56(1):5-6.

4. Luders C, Castro MC, Titan SM, De Castro I, Elias RM, Abensur $\mathrm{H}$, et al. Obstetric outcome in pregnant women on long-term dialysis: a case series. Am J Kidney Dis. 2010;56(1):77-85.

5. Haase M, Morgera S, Bamberg C, Halle H, Martini S, Hocher B, et al. A systematic approach to managing pregnant dialysis patients - the importance of an intensified haemodiafiltration protocol. Nephrol Dial Transplant. 2005;20(11):2537-42.

6. Hold PM, Wong CF, Dhanda RK, Walkinshaw SA, Bakran A. Successful renal transplantation during pregnancy. Am J Transplant. 2005;5(9):2315-7.

7. Davis WD, Ibrahim MA, Hussey JL. Renal transplantation in early pregnancy. Transplantation. 1988;46(1):155-7.

8. Burleson RL, Sunderji SG, Aubry RH, Clark DA, Marbarger P, Cohen RS, et al. Renal allotransplantation during pregnancy. Successful outcome for mother, child, and kidney. Transplantation. 1983;36(3):334-5.

9. Pérgola PE, Kancharla A, Riley DJ. Kidney transplantation during the first trimester of pregnancy: immunosuppression with mycophenolate mofetil, tacrolimus, and prednisone. Transplantation. 2001;71(7):994-7.

10. Miller BW, Howard TK, Goss JA, Mostello DJ, Holcomb WL Jr, Brennan DC. Renal transplantation one week after conception. Transplantation. 1995;60(11):1353-4.

11. Solá R, Ballarin J, Cabero L, Doñate L, del Rio G. Renal transplantation during pregnancy. Transplant Proc. 1988;20(4):720-1.

12. Cabero L, Sola R, Ballarin JA, Esteban-Altirriba J. Renal transplantation during pregnancy-a case report. J Perinat Med. 1988;16(2):149-51.

13. Merlob P, Stahl B, Klinger G. Tetrada of the possible mycophenolate mofetil embryopathy: a review. Reprod Toxicol. 2009;28:105-8. 
14. Sollinger HW. From mice to man: the preclinical history of mycophenolate mofetil. Clin Transplant. 1996;10(1 Pt 2):85-92.

15. Danielsson BR, Danielson M, Rundqvist E, Reiland S. Identical phalangeal defects induced by phenytoin and nifedipine suggest fetal hypoxia and vascular disruption behind phenytoin teratogenicity. Teratology. 1992;45:247-58.

16. Koren G. Mycophenolate mofetil: emerging as a potential human teratogen. Can Fam Physician. 2008;54:1112-3.

17. Jain AB, Reyes J, Marcos A, Mazariegos G, Eghtesad B, Fontes PA, et al. Pregnancy after liver transplantation with tacrolimus immunosuppression: a single center's experience update at 13 years. Transplantation. 2003;76:827-32.

18. Kainz A, Harabacz I, Cowlrick IS, Gadgil S, Hagiwara D. Analysis of 100 pregnancy outcomes in women treated systemically with tacrolimus. Transpl Int. 2000;13(Suppl 1):S299-300.
19. Bar J, Stahl B, Hod M, Wittenberg C, Pardo J, Merlob P. Is immunosuppression therapy in renal allograft recipients teratogenic? A single-center experience. Am J Med Genet A. 2003; 116A(1):31-6.

20. Augustine K, McMahon CL, Pergament E. Organ transplantation and pregnancy. http://www.fetal-exposure.org/resources/wp-content/ uploads/2009/05/organtransplantation_mar2002.pdf. Accessed 30 Jul 2011.

21. Hou SH. Frequency and outcome of pregnancy in women on dialysis. Am J Kidney Dis. 1994;23:60-3.

22. Barua M, Hladunewich M, Keunen J, Pierratos A, McFarlane P, Sood M, et al. Successful pregnancies on nocturnal home hemodialysis. Clin J Am Soc Nephrol. 2008;3:392-6. 